\title{
Thermal effects in highly packed recording media
}

\author{
C.R. Chang.a, I. Klik ${ }^{\mathrm{b}}$, Y.D. Yao ${ }^{\mathrm{b}}$, J.-S. Yang ${ }^{\mathrm{c}}$ \\ 'Departmont of Physics, National Taiwan Unitersity. Taipei 107, Taiwan, ROC \\ 'Institute of Phwics, Academia Sinica, Taipei 115, Taiwan, ROC \\ - Division of General Education, National Taiwan Ocean University, Keelung 202, Taiwan, ROC
}

\begin{abstract}
The dependence of coercivity on packing density in media composed of acicular particles is discussed. We conclude that the nonlinear dependence at small and the linear dependence at moderate packing densities is due to clipolar interactions between the particles; at higher packing densities the bulk magnetization reversal mechanism sets in. It is shown that both the range of linear packing density dependence and the transition to the bulk mode are determined by ambient temperature and driving field frequency. $\subseteq 1998$ Elsevier Science B.V. All rights reserved.
\end{abstract}

Keywords: Coercivity; Fine particles; Relaxation - Néel

The dependence of coercivity on the volume packing density $p$ of particulate media is an old subject which, to date, remains poorly understood since complicated many-body effects are apparently involved. Particles within magnetic media are, as a rule, aggregated and their aggregation leads to mutual interactions whose magnitude is hard to estimate. However, as a rule, the coercivity $H_{\mathrm{c}}(p)$ decreases with the packing density $p$ and this observation is expressed in the heuristic expression [1]

$H_{\mathrm{c}}(p)=H_{\mathrm{c}}^{(\mathrm{b})}+\left[H_{\mathrm{c}}^{(\mathrm{p})}-H_{\mathrm{c}}^{(\mathrm{b})}\right](1-p)$

which interpolates between the bulk coercivity $H_{c}^{(\text {b) }}$ at $p=1$ and the isolated particle coercivity $H_{\mathrm{c}}^{(\mathrm{p})}$ at zero packing density. Physically Eq. (1) corresponds to gradual loss of surface anisotropy which takes place as the particles are being compressed together. Interaction effects between the particles are neglected and so is a possible change in the reversal mechanism: At small packing densities the particles reverse by magnetization rotation while domain walls are presumably present in the bulk.

The interpolation law, Eq. (1), is in reasonably good overall agreement with experimental data but significant deviations from the linear dependence have been

\footnotetext{
*Corresponding author. Fax: +88623639984: e-mail crchang(ajphys.ntu.edu.tw.
}

noted [2] at both small and large packing densities. In particular, particles with large acicularity exhibit a (nonlinear) decrease in coercivity when closely packed [3]. It has been suggested that the nonlinear coercivity behavior at small packing densities results from a nonuniform distribution of particulate aggregates and from a scraping process $[4,5]$ used in the sample preparation. The origin of deviations from linearity at large packing densities remains, so far, unclear though nonlinear $p$-dependence has been found in numerical simulations $[2,6]$.

In the discussion of Eq. (1) we have stated that the interpolation law neglects interactions between the individual particles and that only the loss of their surface anisotropy upon packing is taken into account. However, mutual dipolar interactions beween the particles certainly influence the hysteresis properties of the packed medium and for this reason we address here the dependence of coercivity on the mutual coupling strength. The static Stoner-Wohlfarth limit [7] can, in this regard, be somewhat misleading and we study here, therefore, the more realistic temperature- and frequency-dependent dynamic coercivity derived from a master equation [8,9].

For a general system the probabilistic description is available only in the weak coupling limit [8] and we thus need to restrict ourselves to the simple model of two identical uniaxial particles with mutual dipolar coupling [10] whose thermally activated dynamics are known for all coupling strengths $[8,9]$ if coherent rotation of 


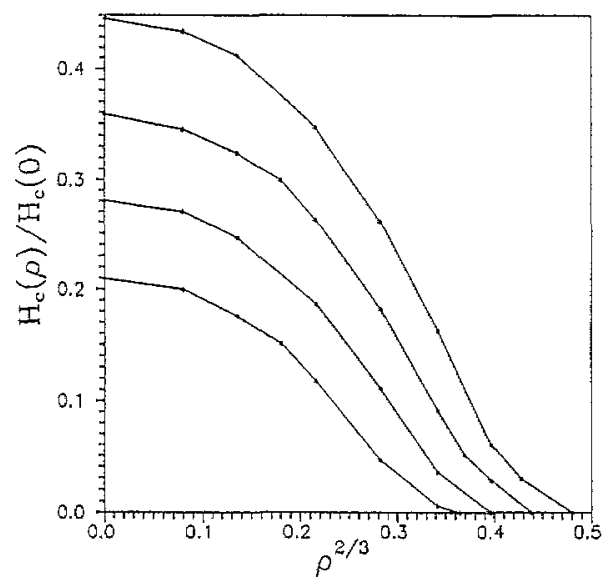

Fig. 1. The coercivity of a particle pair with dipolar interactions favoring antiparallel magnetization orientation (antiferromagnetic coupling with a $90^{\circ}$ bond angle) versus the coupling strength $p^{2 / 3} \sim p$. We chose here the model parameters $K V / k_{\mathrm{B}} T=42$ where $T$ is temperature and $k_{\mathrm{B}}$ the Boltzmann constant. The driving field frequency is $f=10^{3} \mathrm{~Hz}$ (the top-most curve), $10^{1}, 10^{-1}$ and $10^{-3} \mathrm{~Hz}$ (the lower-most curve).

magnetization is assumed. In this model the easy axes of both particles are parallel to the applied field and the case of a $90^{\circ}$ bond angle [8-10] is a rough approximation of a medium composed of aligned highly acicular particles packed somewhat like a bundle of pencils, say. We refer the reader to Refs. $[8,9]$ for details of the probabilistic analysis of the thermally activated dynamics; here we only note that the strength of mutual antiferromagnetic coupling between the particles is given [10] by the dimensionless parameter $\rho=M_{\mathrm{s}}^{2} V / 2 K R^{3}$, where $M_{\mathrm{s}}$ is the saturation magnetization of an isolated particle, $V$ its activation volume and $K$ its anisotropy constant. In our crude-bundle approximation the packing density is inversely proportional to the square of the distance $R$ separating the particles so that

$p \sim \rho^{2 / 3}$.

We assume that the dependence of $p$ on $\rho$ is monotonic. To zero coupling strength there corresponds, obviously, a zero packing density but there must also exist a limiting value of $\rho$ beyond which the particles cannot be compressed without losing their individual identities.
A sample plot of the two-particle coercivity versus the coupling strength parameter $\rho^{2 / 3}$ is shown in Fig. 1. It is seen that in the medium range of coupling strengths the coercivity dependence on $p$ is approximately linear but that deviations from linearity occur at both small and large coupling strengths, with the small $\rho$ deviations being particularly prominent at low driving frequencies or high ambient temperatures. High coupling strengths stabilize the antiparallel states $\uparrow \downarrow$ and $\downarrow \uparrow$ and coercivity tends to zero. We, therefore, propose that at small packing densities the hysteresis properties of the medium are determined by mutual interparticle interactions and that the resulting $p$-dependence is of the type depicted in Fig. 1, i.e., it is linear at moderate packing densities and nonlinear at $p \ll 1$. At higher packing densities the bulk reversal mechanism sets in within densely agglomerated regions of the sample; this mechanism gives rise to a different dependence of coercivity on $p$. We stress again that the transition region between the two mechanisms depends not only on packing density, but also on temperature and frequency at which the coercivity is measured via the finite probability of nucleating and unpinning a domain wall [11]. Moreover, our plot of Fig. I shows that the transition beween linear and nonlinear $p$-dependence at small and moderate packing densities (where the particulate reversal mechanism dominates) is frequency and temperature dependent as well.

\section{References}

[1] L. Néel, Comp. Rend. 234 (1947) 1150.

[2] J.E. Knowles, IEEE Trans. Magn. 21 (1985) 2576.

[3] S. Umeki, H. Sugihara, Y. Taketoni, Y. Imaoka, IEEE Trans. Magn. 17 (1981) 3014.

[4] C.R. Chang, J.P. Shyu, J. Magn. Magn. Mater. 120 (1993) 197.

[5] C.R. Chang, J.P. Shyu, J. Appl. Phys. 73 (1993) 6659.

[6] F.J.A. den Broeder, W. Hoving, P.J.H. Bloemen, J. Magn. Magn. Mater. 93 (1991) 562.

[7] S. Nieber, H. Kronmüller, Phys. Stat. Sol. (a) 165 (1991) 503.

[8] I. Klik, C.R. Chang, Phys. Rev. B 52 (1995) 3540.

[9] I. Klik, J.S. Yang, C.R. Chang, J. Appl. Phys. 76 (1994) 6588.

[10] W. Chen, S. Zhang, H.N. Bertram, J. Appl. Phys. 71 (1992) 5579.

[11] I. Klik, C.R. Chang, J, Magn. Magn. Mater. 114 (1992) L235. 